\title{
Evaluación del Programa de Inclusión y Alfabetización Digital de México con el modelo CIPP
}

\author{
Inés Domínguez Domínguez ${ }^{a}$, Dora Luz González-Bañales ${ }^{b}$ \\ ${ }^{a}$ Doctorado en Ciencias de la Educación, Instituto Universitario Anglo Español, Durango, México, \\ airamseni81@gmail.com \\ ${ }^{\mathrm{b}}$ Departamento de Sistemas y Computación, Instituto Tecnológico de Durango, Tecnológico Nacional \\ de México, Durango, México.doraglez@itdurango.edu.mx
}

\section{Resumen}

En México se tiene más de 20 años intentando implementar en las instituciones educativas a nivel de educación básica programas que incorporen el uso de las TIC en las aulas, sin embargo los programas implementados no han sido permanentes y cada seis años se modifican, tal inestabilidad se ha debido a que en parte estos programas se evalúan más desde los números que desde los impactos reales para que sean eficientes. La investigación que se realizó tuvo como objetivo general evaluar el Programa de Inclusión y Alfabetización Digital(PIAD) en una Escuela Primaria del Municipio de Durango, Estado de Durango, México, principalmente con el programa de entrega de tabletas a niños de quinto grado de primaria. Los participantes fueron tres grupos con un total de 107 estudiantes, 59 padres de familia, tres profesores responsables de implementar el programa y la directora de la escuela. Para evaluar el programa se utilizó el Modelo CIPP (Contexto, Insumo, Proceso y Producto) propuesto por Stufflbeam. Algunos de los resultados más sobresalientes son: En lo referente a la dimensión Insumo los estudiantes valoraron que la institución educativa les informó acerca de su responsabilidad en el cuidado de la tablet, además de que su salón tuviera las condiciones necesarias para realizar las actividades propuestas por el profesor. En cuanto a la dimensión del proceso mencionaron que cumplieron las actividades tanto con sus compañeros, como de forma individual, sin embargo, muy pocas veces publicaron o compartieron sus experiencias o producciones multimedia. En la dimensión Producto, de lo que más destacaron fue que al término del ciclo lograron hacer uso de la tecnología de forma responsable cuidando su información personal y la de los demás, pero no lograron publicar y compartir ideas y opiniones mediante diversos productos digitales (audio, vídeo, texto, fotografia). 


\section{Palabras clave: Alfabetización Digital, CIPP, inclusión digital}

\section{Introducción}

Actualmente el uso de las Tecnologías de Información y Comunicación (TIC) se ha vuelto indispensable para sectores como el agrícola, industrial, económico, salud, educativo entre otros. En México, en cuanto al ámbito educativo, las TIC se hacen presente en todos los niveles educativos desde preescolar hasta posgrado, y aunque, cada vez son más las escuelas que cuentan con infraestructura tecnológica que permite tanto a docentes como a estudiantes acceder a un sinfín de recursos didácticos, informativos y recreativos. El gobierno mexicano (2012- 2018) continúa en la búsqueda de consolidar el uso de las TIC dentro de las aulas como parte del proceso enseñanza - aprendizaje que contribuyan a que tanto niños como jóvenes estén preparados para la vida digital, ya que como se puede leer en (SEP, Secretaría de Educación Pública, 2011, pág. 64):

"la ausencia de una política de tecnologías de la información y la comunicación en la escuela pública aumenta la desigualdad entre los países y las personas. La Organización de las Naciones Unidas para la Educación, la Ciencia y la Cultura (Unesco) prevé que construir sociedades del conocimiento contribuye a los Objetivos de Desarrollo del Milenio".

De modo que en el 2013 la Secretaría de Educación Pública (SEP) dio inicio al Programa de Inclusión y Alfabetización Digital (PIAD) el cual busca:

fortalecer el sistema educativo mediante la entrega de dispositivos personales, promoviendo la reducción de la brecha digital y el uso de las TIC en el proceso enseñanzaaprendizaje, fomentando la interacción entre alumnos, docentes y padres de familia, y fortaleciendo el aprendizaje de los alumnos de las escuelas públicas, con el objetivo de reducir el rezago educativo. (Digital, 2014).

De la literatura revisada, a manera de introducción, se resume que la mayoría de las investigaciones que se han realizado en diferentes países van orientadas a medir la eficiencia de los programas que implican el uso de las TIC como una herramienta para la mejora del aprendizaje en los niveles de educación básica, y generalmente fueron solicitados por Centros Educativos de Educación o Secretarías de Educación en diversos países a través de Institutos de Investigación en donde han participado varios investigadores (Alvarado, 2010; Centro de Altos Estudios e Investigaciones Pedagógicas y administrado por el Colegio de Estudios Científicos y Tecnológicos del Estado de Nuevo León (CECyTE, NL), 2005; Centro Anáhuac de Investigación, Servicios Educativos y Posgrado de la Facultad de Educación de la Universidad Anáhuac, 2005; Centro de Investigación Educativa y Actualización de Profesores A.C., 2005; Di et al., 2012; Facultad 
Latinoamericana de Ciencias Sociales (Flacso), sede México, 2007; García, 2007; Gioia de Melo et al., 2013; Holland et al., 2006; Instituto Latinoamericano de la Comunicación Educativa, 2004; Instituto Latinoamericano de la Comunicación Educativa, 2005; Ministerio de Educación, 2011; Ministry of Education and Human Resources Development Solomon Islands Government, 2010; Nussbaum, 2011; Sánchez \& Martínez, 2012; Santiago et al., 2010; Secretaría de Educación Pública, 2007; Secretaría de Educación Pública, 2008, Secretaría de Educación Pública, 2009; Universidad de Guadalajara, 2006).

\section{Investigación evaluative}

El diseño metodológico de una investigación evaluativa corresponde al momento en el cual se deben tomar las decisiones correspondientes a las estrategias o actividades que debe realizar el evaluador para el logro de los objetivos de evaluación o la respuesta a las preguntas de evaluación por él planteadas. En la figura 1 se presentan las etapas de la metodología de investigación evaluativa (Correa, Puerta, \& Restrepo, 2002).

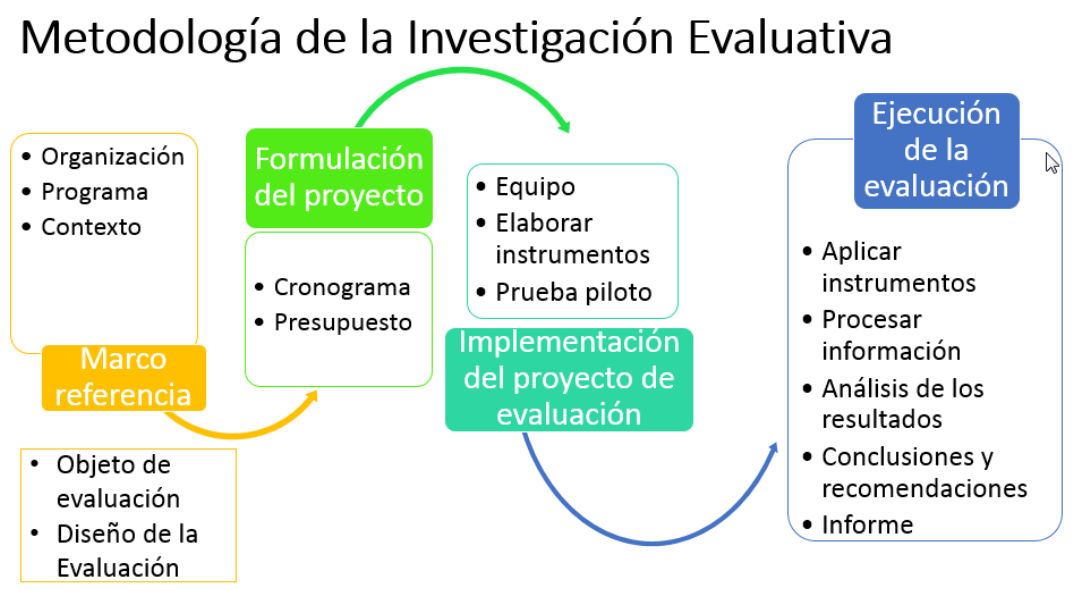

Fig 1. Etapas de la metodología de investigación evaluativa Fuente: (Correa, Puerta, \& Restrepo, 2002)

Como se puede observar, el diseño metodológico consta te cuatro etapas claramente identificadas, la primer etapa se llama marco de referencia en donde lo primero que se hace es obtener todo la información sobre el objeto a evaluar, para que posteriormente se pueda definir el objeto y diseño de la evaluación. Durante la segunda etapa se formula el proyecto, 
es decir la evaluación a realizar. En lo que respecta a la tercera etapa ésta consiste en la implementación del proyecto de evaluación y por último la etapa cuatro se lleva a cabo la ejecución de la evaluación.

Según Boruch y Wortman (1979) en (Bausela, 2003) no existe un modelo generalizado de investigación evaluativa, y a lo largo de los años desde 1975 diferentes autores han propuestos distintos modelos de evaluación, uno de ellos es precisamente el modelo CIPP propuesto por Daniel Stufflebeam en 1987 y que a la fecha sigue vigente, pues autores como Bausela, Esperanza (2003), Damián, Javier \& Montes, Eusebio (2011), Guerrero, Daniel (2011) continúan citando su modelo. La metodología de dicho modelo se puede revisar en (Correa, Puerta, \& Restrepo, 2002, pág. 98), este modelo también plantea cuatro niveles o etapas, las cuales se pueden observar en la figura 2.

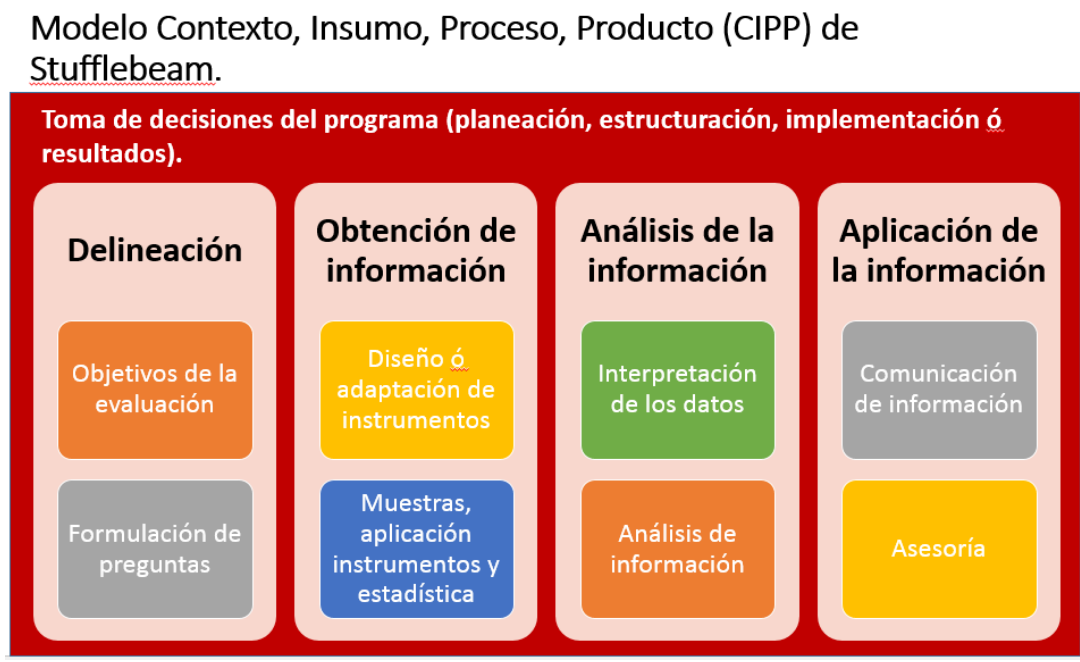

Fig. 2 Metodología del Modelo CIPP

Fuente: Elaboración propia a partir de (Correa, Puerta, \& Restrepo, 2002).

\section{Marco de referencia}

Como parte de la política educativa y con la finalidad de alcanzar los objetivos de la incorporación de las TIC en el ámbito educativo, la Secretaría de Educación Pública de México (SEP) dio inició con modelo 1 a 1 en el 2013, en 2015 - 2016 este modelo recibió el nombre de Programa de Inclusión y Alfabetización Digital (PIAD) el cual busca: 
Fortalecer el sistema educativo mediante la entrega de dispositivos personales, promoviendo la reducción de la brecha digital y el uso de las TIC en el proceso enseñanza-aprendizaje, fomentando la interacción entre alumnos, docentes y padres de familia, y fortaleciendo el aprendizaje de los alumnos de las escuelas públicas, con el objetivo de reducir el rezago educativo (Mèxico Digital, 2014).

Hasta el año 2015 fueron 15 los Estados de la República Mexicana que participan en el PIAD, a los cuales se les entregaron laptops y tablet a niños de quinto y sexto año de primaria de escuelas públicas. A continuación, se mencionan los Estados de la República Mexicana que desde 2013 implementaron el PIAD.

En 2013 se inició con Sonora, Colima y Tabasco, para el 2014 se incluyó a Distrito Federal, México y Puebla, por último, en 2015 se agregaron a la lista Chihuahua, Durango, Hidalgo, Nayarit, Quintana Roo, Tlaxcala, Sinaloa, Yucatán y Zacatecas (Subsecretaría de Educación Pública de México, 2014).

Es importante resaltar que el equipo entregado:

- Es propiedad de los estudiantes.

- Tiene dos años de garantía por fallas de fabricación.

- Es responsabilidad de los estudiantes y padres de familia.

- En caso de robo o extravío, no será repuesto. (Subsecretaría de Educación Básica de México, 2013).

Se hablará del PIAD específicamente en el Estado de Durango, puesto que fue en la ciudad capital de éste donde se llevó acabo esta investigación. La coordinación del PIAD estuvo a cargo de la Dirección de Informática Administrativa (DIA) de la Secretaría de Educación del Estado de Durango (SEED). Durante el ciclo escolar 2015 - 2016 se entregaron 39200 tablet a niños que cursaban el quinto año de primaria en un total de 2035 escuelas públicas, con un total de 2719 grupos.

\section{Objetivos}

\subsection{Objetivo General}

- Evaluar los resultados del Programa de Inclusión y Alfabetización Digital en la Escuela Primaria Anexa del municipio de Durango, mediante el modelo de evaluación CIPP. 


\subsection{Objetivos específicos}

- Determinar si los recursos humanos y materiales del PIAD fueron los adecuados para llevar a cabo el programa (Insumo).

- Identificar posibles discrepancias entre el diseño final establecido para el PIAD y su implementación real, desde la valoración de alumnos, profesores y padres de familia (Proceso).

- Identificar hasta qué punto el PIAD alcanzó los objetivos para lo cual fue creado (Producto).

\section{Metodología}

\subsection{Selección de enfoque de investigación}

En la revisión bibliográfica realizada se observó que en México poco se ha publicado sobre investigaciones evaluativas aplicadas al área de programas TIC en educación básica, sin embargo, como se menciona en (Correa, Puerta, \& Restrepo, 2002) "La investigación evaluativa se vale de los métodos y el instrumental de la investigación social; por lo tanto, su desarrollo sigue sus mismas evoluciones, lo cual le permite una aproximación permanente a criterios de cientificidad" (Pág. 11).

En el caso de la presente investigación se siguió el modelo de evaluación de Daniel Stufflebeam conocido como CIPP por sus siglas de Contexto, Insumo, Proceso y Producto, y el cual sigue siendo vigente para la evaluación de programas. Como se menciona en (Torres Maldonado, 2015) el modelo CIPP pretende mejorar el programa que se evalúa y no solo resaltar sus debilidades.

\subsection{Alcance de la investigación}

El alcance de la investigación es de tipo exploratorio y descriptivo. Exploratorio, porque como mencionaron (Hernández Sampieri, Fernández Collado, \& Baptista Lucio, 2006) "Los estudios exploratorios se realizan cuando el objetivo es examinar un tema o problema de investigación poco estudiado, del cual se tienen muchas dudas o no se ha abordado antes" (pág. 141), en este sentido, a la fecha en que se decidió realizar la investigación evaluativa del programa PIAD, no se encontraron evidencias de otras investigaciones a éste programa en la ciudad de Durango, Duango, de modo que se exploró acerca del programa para poder determinar el contexto en que éste se presentó. Al mismo tiempo el alcance fue 
descriptivo, ya que se revisaron las cuatro dimensiones del modelo CIPP, para describir cómo fue la implementación del PIAD en cuanto al Contexto, Insumo, Proceso y Producto. Fue una investigación no experimental y transversal ya que la recolección de información se hizo en solo momento.

\subsection{Diseño metodológico}

El diseño metodológico que se empleó en la investigación hace uso de técnicas cuantitativas y cualitativas, como se muestra en la tabla1.

Tabla 1. Empleo de técnicas cuantitativas y cualitativas

\begin{tabular}{lll}
\hline \hline & Cuantitativa & Cualitativa \\
\hline Contexto & & Revisión de Documentos \\
Insumo & Cuestionario & Grupo Focal \\
Producto & Cuestionario & Revisión de Documentos \\
Proceso & Cuestionario & GRupo Focal \\
\hline \hline
\end{tabular}

Fuente: Elaboración propia

\subsection{Población}

En el municipio de Durango (México) para el ciclo escolar 2015 - 2016 se entregaron un total de 12, 256 tablets en 377 escuelas, 182 fueron primarias estatales y 195 primarias federales, en las primeras escuelas se entregaron 6,125 tablets a los alumnos y 441 a profesores, mientras que en las federales se entregaron 5, 257 tablets a los alumnos y 433 a los profesores.

\subsection{Método de muestreo}

La selección de la escuela donde se desarrolló la investigación fue de tipo no probabilístico, empleado el muestro por conveniencia.

Se eligió la Escuela Primaria Anexa de la ciudad de Durango del Sector 1 perteneciente a la Zona 15 de escuelas primarias estatales, en donde participaron 107 alumnos de sexto año distribuidos en tres grupos, de los cuales 56 fueron hombres y 51 mujeres, la edad de los participantes oscila entre los 11 y 13 años; se tuvo la participación de 59 padres de familia de los alumnos de sexto año, de los cuales 17 fueron hombres y 42 mujeres, la edad promedio de los padres de familia es de 39 años; se solicitó la colaboración de los tres profesores (2 mujeres y 1 hombre) de quinto año quienes fueron los encargados de 
implementar el programa PIAD con los alumnos el ciclo escolar 2015 -2016, cuando estaban en quinto grado.

\subsection{Recolección de datos}

La recolección de datos se realizó mediante entrevista a profesores y estudiantes, a través de un grupo focal, cuestionarios a profesores, estudiantes, padres de familia, así como la revisión de documentos acerca del programa PIAD. El número de profesores entrevistados fueron los tres profesores encargados de implementar el programa, esta entrevista se realizó antes de la aplicación de cuestionarios. Además, se entrevistó a un estudiante de cada grupo, después de que respondieron el cuestionario.

\subsection{Diseño de cuestionario}

En cuanto a los cuestionarios que se emplearon para la recolección de datos, estos se diseñaron considerando el documento base del programa emitido por Secretaria de Educación Pública en donde se establecen los objetivos del programa y las competencias que los estudiantes deben alcanzar mediante el uso de los contenidos digitales incorporados en la tablet, además de que se alineo a las fases del modelo de evaluación. Fueron tres cuestionarios uno para profesores, otro para estudiantes, y un último para padres de familia.

\subsubsection{Cuestionario para estudiantes}

El cuestionario para estudiantes tuvo un total de 66 ítems, de los cuales 24 fueron dicotómicos y 42 de escala tipo Likert empleando en algunos (1. En desacuerdo, 2. Parcialmente en desacuerdo, 3. Parcialmente de acuerdo, 4. De acuerdo) y en otros ítems (1. Nunca, 2. Casi Nunca, 3. Casi siempre, 4. Siempre), estos 43 ítems estuvion divididos en cinco secciones relacionadas con las dimensiones del modelo CIPP y una sección general con el objetivo de obtener datos del participante.

La sección II contiene nueve ítems, los cuales tienen que ver con los Insumos proporcionados a los estudiantes, esta sección busca indagar sobre si el estudiante conocía los elementos o instrucciones del uso de la tablet, así como, si los insumos empleados para el funcionamiento del programa eran los adecuados $(\alpha=0.624)$. La sección III está relacionada con el Proceso del modelo CIPP, compuesta de 17 ítems de escala tipo Likert $(\alpha=0.775)$ y 6 ítems dicotómicos. Estos ítems miden la funcionalidad de los accesos a material didáctico con lo que contaba la tablet y la operatividad técnica de la tablet durante 
todo el tiempo que se implementó el programa. La sección IV contiene un total de 13 ítems $(\alpha=0.838)$ de escala tipo Likert, en esta sección se mide los resultados al final del ciclo escolar en los aspectos de aprender a aprender y aprender a convivir, los cuales se enmarcaron como objetivos a lograr con el programa, dentro del documento base del PIAD, esta sección se relaciona con el Producto del modelo de evaluación CIPP. Finalmente, la sección V cuenta con solo tres ítems también de escala tipo Likert ( $\alpha=0.579)$, esta sección pretende medir que tanto se lograron los objetivos del PIAD en cuanto a la motivación del alumno para asistir a clases, en cómo fue la enseñanza del profesor y sí lo aprendido lo compartieron con su familia, todo esto originado por el uso de la tablet dentro del programa.

\section{Resultados}

\subsection{Insumo}

En cuanto a la información que se proporción a los estudiantes, según los resultados, éstos supieron del proceso de la entrega de su Tablet, de su responsabilidad del cuidado de ésta, así como de las secciones que contenían y que sus dudas fueron siempre aclaradas, además de que la energía eléctrica siempre estuvo disponible, lo único que comentó la mayoría es no haber sido informados sobre el sitio web de micompu.mx para su consulta.

Hay que destacar la importancia del quehacer docente, que, si bien el documento base acerca del programa no mencionaba que se estableciera un reglamento de uso y de cuidado de la tablet, la institución con la colaboración de los profesores realizó un reglamento del uso y cuidado de la tablet, el cual se entregó a los estudiantes y debían firmar los padres de familia.

\subsection{Proceso}

En cuanto a el tiempo que los estudiantes, hicieron uso de la tablet en el aula, según los resultados obtenidos se puede rescatar que solo el 50.5\% de los estudiantes reconocieron que el profesor llevó a cabo distintas actividades mediante el uso de la tablet. Sin embargo, más del $70 \%$ de los estudiantes comentaron que el profesor fomentó el trabajo en equipo a través de la tablet.

En cuanto a las secciones que los estudiantes definieron como las que más utilizaron fueron Planea y Busca, mediante las cuales realizaron actividades escolares, recreativas y 
familiares, así como consulta de vídeos interactivos, mapas, audiolibros y libros electrónicos.

Por otra parte las secciones a las que nunca o casi nunca accedieron fueron Organiza, Exprésate, Colabora, Comparte, Colecciones, Portafolio electrónico y Sitios, a lo que quizás esto tenga relación con la conexión a Internet, ya que como lo mencionaron los profesores el acceso a él, no fue muy bueno, por lo que tal vez este haya sido uno de los factores del por qué los estudiantes no accedieron a estas secciones.

En lo que se refiere a si durante el PIAD los estudiantes llevaron su tablet con la batería cargada al $100 \%$ más del $80 \%$ de ellos afirmaron que siempre lo hicieron, por otra parte, menos del $60 \%$ de ellos reconoció que su tablet estuvo en buenas condiciones, pues siguió las instrucciones de su cuidado.

De las fallas más comunes que los estudiantes mencionaron fueron: el acceso a Internet y que la tablet estaba lenta. Además el 76.6\% de los estudiantes dijeron que nunca recibieron la visita del director en sus aulas para saber acerca de los avances del PIAD y el $43 \%$ de los estudiantes dijo que enseñó a quienes viven en su casa a utilizar las aplicaciones, contenidos, recursos y a navegar por Internet desde su tablet.

Asimismo, 71\% de los estudiantes dijo que utilizó Internet en la escuela solo para buscar información. Y mencionaron que las materias en donde más utilizaron la tablet fueron: español y matemáticas.

\subsection{Producto}

Los resultaron sugieren que entre el 50\% y el 70\% de los estudiantes lograron adquirir los distintos aprendizajes planteados en los objetivos del programa, uno en mayor medida que otros, además los estudiantes reconocen saber organizar, clasificar, buscar, consultar, seleccionar información en distintos formatos a través de distintas páginas web, así como que fueron capaces de auto planear sus propias tareas o actividades e identificar el funcionamiento de distintas aplicaciones, así como desarrollar habilidades para resolver problemas.

A su vez el $60.7 \%$ de los estudiantes dijo que casi siempre o siempre la forma de enseñanza de su profesor a través del uso de la tablet, les motivó a asistir a clases y que además el uso de la tablet les permitió mejorar sus aprendizajes.Sin embargo, queda la pregunta ¿Y el otro $50 \%$ o $30 \%$ de los estudiantes?, ¿qué paso con ellos, por qué no lograron alcanzar los objetivos planteados? 


\section{Conclusiones}

En lo referente al insumo en la parte de los estudiantes se destaca la importancia de informarles a ellos sobre el proceso de la entrega de la tablet, de su responsabilidad del cuidado de ésta, así como de las secciones que contiene su material de trabajo y aclarar sus dudas. Por el lado de los padres de familia. un elemento que resulta importante es la constante y adecuada comunicación entre ellos y los profesores, a fin de estar enterados del proceso de entrega de la tablet y de la notificación de su responsabilidad del cuidado de la misma. Y finalmente para los profesores en la parte de insumo los profesores mencionaron que la información que se les proporcionó en el curso de capacitación acerca del funcionamiento del PIAD no fue clara. y que además el número de horas asignadas para la capacitación, no fueron suficientes.

Por otro lado en el tema de Proceso los estudiantes resaltaron la labor del profesor, sobre todo en la forma que llevó a cabo distintas actividades mediante el uso de la tablet, incluyendo el fomentar el trabajo en equipo, a pesar de que las secciones a las que nunca o casi nunca accedieron fueron Organiza, Exprésate, Colabora, Comparte, Colecciones, Portafolio electrónico y Sitios, a lo que quizás esto tenga relación con la conexión a Internet, ya que como lo mencionaron los profesores el acceso a él, no fue era muy bueno, por lo que tal vez este haya sido uno de los factores del por qué los estudiantes no accedieron a estas secciones. Para los padres de familia, se destaca que su apoyo es importante para lograr el uso eficiente de la tablet como recurso para su aprendizaje. Asimismo, los padres de familia destacaron que al realizar con sus hijos las actividades con la tablet les permitió aprender junto con ellos, lo que significa que los niños pueden alfabetizar digitalmente a sus padres. Por otro lado, los profesores expresaron su descontento con el proceso de capacitación y seguimiento del programa, ya que para ellos no basta solo recibir la información teórica, requieren que se les diga cómo diseñar estrategias didácticas mediante el uso diferentes tecnologías y dispositivos como la tablet. A su vez para ellos resulta importante tener un acompañamiento que les permita retroalimentar sus dudas.

Finalmente para el Producto se destaca que los estudiantes lograron adquirir los distintos aprendizajes planteados en los objetivos del programa, los estudiantes reconocieron saber organizar, clasificar, buscar, consultar, seleccionar información en distintos formatos a través de distintas páginas web, además de que fueron capaces de auto planear sus propias tareas o actividades e identificar el funcionamiento de distintas aplicaciones, así como desarrollar habilidades para resolver problemas. Por toro lado, los padres de familia reconocieron que sus hijos pudieron investigar en Internet con su tablet de manera 
consiente y ágil, y que fueron capaces de comunicarse y elaborar proyectos con otros niños de su edad a través del uso de su tablet, así como conocer el funcionamiento y concepto de las TIC para usarlo a favor de su aprendizaje e identificaron la importancia de tener cuidado sobre la información que comparten en redes sociales o alguna página de Internet. Finalmente los profesores expresaron que la mayoría de sus estudiantes logró organizar, clasificar y transportar información y archivos en diferentes formatos.

A manera de cierre de conclusiones, se puede decir que, para los estudiantes de éste siglo XXI, es importarte la interacción en el aula a través del uso de las TIC, en donde pueden tener acceso a un sinfín de aplicaciones educativas y lúdicas que les ayuden en su aprendizaje, sin lugar a dudas el papel del docente sigue y segurá siendo esencial para mediar, guiar y facilitar el aprendizaje de los estudiantes, pero para ello, es indispensable que el docente tenga las habilidades digitales que le permitan emplear herramientas tecnológicas como apoyo a sus estrategias de enseñanza. Aunado a lo anterior, es también necesario que las autoridades educativas adquieran el compromiso de apoyar a las instituciones educativas, proveyéndolas de la infraestructura física y tecnológica necesaria y de calidad que permita la incorporación de las TIC, así como de capacitación pertinente a profesores y directivos. Asimismo, la participación de los padres de familia sigue siendo crucial, pues ellos también debieran contar con habilidades digitales que les permita apoyar a sus hijos, para que hagan buen uso de las TIC en casa y en la escuela.

\section{Referencias}

Bausela, E. (2003). Metodología de la Investigación Evaluativa: Modelo CIPP. Revista Complutense de Educación, 361- 376.

Correa, S., Puerta, A., \& Restrepo, B. (2002). Especialización en teoría, métodos y técnicas de investigación social. Investigación Evaluativa. Bogotá, Colombia: ARFO Editores e Impresores Ltda.

Digital, M. (2014). México Digital. Obtenido de http://www.gob.mx/mexicodigital/articulos/programa-de-inclusion-y-alfabetizaciondigital-piad

Hernández Sampieri, R., Fernández Collado, C., \& Baptista Lucio, P. (2006). Metodología de la Investigación. México, D.F.: McGraw-Hill Interamericana. 
Mèxico Digital. (2014). México Digital. Obtenido de

http://www.gob.mx/mexicodigital/articulos/programa-de-inclusion-y-alfabetizaciondigital-piad

SEP. (2011). Secretaría de Educación Pública. Obtenido de http://www.curriculobasica.sep.gob.mx/images/PDF/planestudios11.pdf

Subsecretaría de Educación Básica de México. (2013). Primariatic. Obtenido de http://www.basica.primariatic.sep.gob.mx/descargas/TIC_DOTACION_BAJA.pdf

Subsecretaría de Educación Pública de México. (2014). Programa de Inclusión y Alfabetización Digital. México, D.F.: SEP.

Torres Maldonado, J. A. (2015). Evaluacion del programa de Ingeniría de Sistemas de la Universidad Cooperativa de Colombia Seccional Bogotá mediante el modelo de evaluación CIPP. San Sebastian, País Vasco, Colombia. 